\title{
A Search for Factors that Predict the Efficacy of Viscosupplementation in Knee Osteoarthritis
}

\author{
R. Michelle Koolaee ${ }^{1,2}{ }^{*}$, H. Ralph Schumacher ${ }^{1}$, Michael Shoemaker $^{3}$, Sarah Mooar ${ }^{1}$, \\ Sally Pullman-Mooar ${ }^{1}$ \\ ${ }^{1}$ Division of Rheumatology, Philadelphia Veterans Affairs Medical Center and Perelman School of Medicine at \\ the University of Pennsylvania, Philadelphia, PA, USA \\ ${ }^{2}$ Division of Rheumatology, Keck School of Medicine at the University of Southern California, Los Angeles, USA \\ ${ }^{3}$ Grand Valley State University Department of Physical Therapy, Grand Rapids, MI, USA \\ Email: *michellekoolaee@gmail.com
}

Received 16 May 2014; revised 25 June 2014; accepted 5 August 2014

Copyright (C) 2014 by authors and OALib.

This work is licensed under the Creative Commons Attribution International License (CC BY).

http://creativecommons.org/licenses/by/4.0/

(c) (i) Open Access

\section{Abstract}

Objective: Previous research demonstrates inconsistent effects of hyaluronate (HA) viscosupplementation on patients with knee osteoarthritis $(\mathrm{OA})$. The purpose of this study was to evaluate factors that predict clinical response to a single intra-articular injection of Hylan GF-20. Methods: This was an observational study of 55 patients with knee $0 A$, scheduled to receive intra-articular injections of Hylan GF-20. These patients met the institution's guidelines for use of viscosupplementation, which entails failure/intolerance of medical management of $\mathrm{OA}$. At baseline, patients completed a series of questionnaires, including the Knee Injury and Osteoarthritis Outcome Score (KOOS) and Patient Health Questionnaire (PHQ-9) depression score. Questionnaires were repeated at three months post-injection. A clinical responder was someone with a change in KOOS score which exceeded the mean minimal detectable change (MDC) values calculated based on test-retest reliability coefficients reported in four prior studies. Hypothesized predictors of response included PHQ-9 score, baseline visual analog scale (VAS) pain score, age, body mass index (BMI), and Kellgren score. Results: There were 35 responders and 20 non-responders. There were no statistically significant differences between responders and non-responders for any of the primary predictors. There were no differences for secondary predictors, including history of knee injury or smoking, prior HA injection, prior intra-articular corticosteroid injection, or location of $0 A$. There was a moderate negative correlation between age and change in total KOOS score $(r=$ $-0.32, p=0.01$ ). Conclusions: Our research did not confirm previous predictors of response to hyaluronate injections, and highlighted the need for prospective studies in order to answer this question.

\footnotetext{
${ }^{*}$ Corresponding author.
}

How to cite this paper: Koolaee, R.M., Schumacher, H.R., Shoemaker, M., Mooar, S. and Pullman-Mooar, S. (2014) A Search for Factors that Predict the Efficacy of Viscosupplementation in Knee Osteoarthritis. Open Access Library Journal, 1: e700. 


\title{
Keywords
}

\section{Hyaluronic Acid, Viscosupplementation, Osteoarthritis, Knee Osteoarthritis, Efficacy, Hyaluronate}

\author{
Subject Areas: Clinical Trials, Rheumatology
}

\section{Introduction}

Hyaluronate (HA) viscosupplementation is one of the treatments for knee osteoarthritis (OA). HA is a critical component of normal synovial fluid and is necessary for joint homeostasis [1]. Osteoarthritic synovial fluid demonstrates a marked decrease in the concentration and molecular weight of HA [1] [2]. These alterations lead to dramatically poorer viscous and elastic properties of synovial fluid, and thereby distort normal joint mechanics [1]. There are several proposed benefits of viscosupplementation on cartilage and synovium, based on both in-vitro and animal studies. These include decreased synovial inflammation, direct analgesic effects, and prevention of further chondrocyte degradation [3]-[6].

Hylan GF-20 (Synvisc $\left.{ }^{\circledR}\right)$ is a high molecular weight HA derivative $\left(6 \times 10^{6}\right.$ Daltons) obtained by reticulation of HA from rooster combs. The initial treatment schedule in knee osteoarthritis consisted of three intra-articular injections of $2 \mathrm{~mL}$ Hylan GF-20 at 1-week intervals. In 2009, Synvisc-One ${ }^{\circledR}$ was approved for a single $6 \mathrm{~mL}$ intra-articular injection treatment regimen given every 6 months. This schedule was effective in two controlled studies versus placebo [7] [8]. Hylan GF-20 was approved in Europe in 1995 and in the United States in 1997 as a medical device. Given their high cost, HA injections are currently offered at the Philadelphia VA Medical Center (PVAMC) when treatments such as oral analgesics and intra-articular corticosteroids (ICS) are ineffective.

The efficacy of HA injections is controversial and a topic of debate. A Cochrane review of 76 trials in 2006 found improvements in pain and function with HA [9], yet a recent review of 89 trials concluded otherwise, with criticisms concerning the quality of the trials [10]. The authors of the present study have clinically observed that some patients appear to respond well to injections, while others do not. Unfortunately, there are few studies investigating how to select patients who are most likely to benefit from HA. In an effort to most effectively utilize this costly therapy, the objective of this study was to look for factors that may predict clinical response to a single intra-articular injection of Hylan GF-20 (Synvisc-One®).

\section{Methods}

This was an observational study of 55 subjects at the PVAMC with knee osteoarthritis. The study protocol was approved by the institutional review board at the PVAMC.

\subsection{Participant Selection}

Participants had OA diagnosed by either a rheumatologist or an orthopedic surgeon, and were already scheduled to receive viscosupplementation with Hylan GF-20 (Synvisc-One ${ }^{\circledR}$ ) based on PVAMC formulary guidelines. These guidelines required failure or intolerance of oral analgesics, physical therapy/brace support, weight loss and/or ICS injections. Patients with a history of inflammatory arthritis were excluded.

\subsection{Study Design}

Figure 1 summarizes the study design. Subjects were given informed consent the day that they were scheduled to receive their HA injection, at either the rheumatology or orthopedic surgery clinic. At that time, a physical examination of the target knee was performed by the treating provider. During the same visit, subjects completed the Knee Injury and Osteoarthritis Outcome Score (KOOS) and the nine-item Patient Health Questionnaire depression score (PHQ-9). The sequestionnaires are described below (see "Measurement Tools"). In addition, all subjects completed an initial assessment form, which included information about medical history, demographics, previous knee injury, and other previous treatments for knee OA. All subjects were required to have a weight bearing radiograph of the target knee within the previous two years. At three months post-injection, 


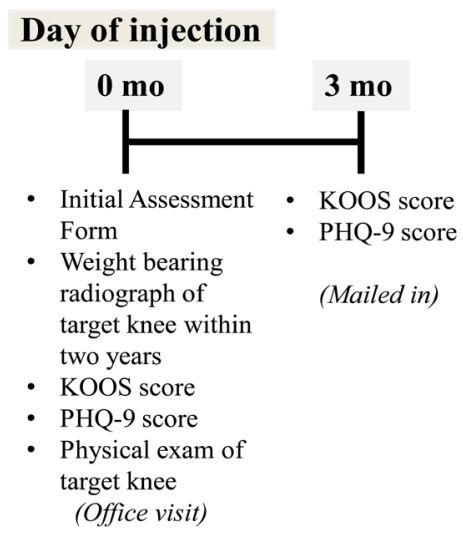

Figure 1. Study design.

subjects were asked to complete and return (by mail) the KOOS and PHQ-9. A series of letters and reminders were used to assure that the three-month forms were completed.

\subsection{Measurement Tools}

Two rheumatologists independently determined the radiographic severity of knee osteoarthritis based on the Kellgren-Lawrence scoring system [11]. Score discrepancies were reviewed jointly to arrive at a final score. The KOOS is a 42-item questionnaire that is used to assess patients' opinion about their knee osteoarthritis. It scored on a zero to 100 scale, with zero representing the most severe symptoms. In addition to an overall score, the KOOS includes subscale scores for symptoms, pain, function (activities of daily living), sports/recreational activities, and quality of life. The questions are identical to the Western Ontario and McMaster Universities Osteoarthritis Index (WOMAC), with the addition of the sports/recreational activities and quality of life sections. It is a valid, reliable, and responsive outcome measure compared to the WOMAC [12] [13]. The PHQ-9 depression score is a nine-item, validated, self-administered questionnaire that is widely used to assess for the presence and severity of depression [14].

\subsection{Hypothesized Clinical Predictors}

The primary hypothesized clinical predictors of clinical response included Kellgren score, age, body mass index (BMI), PHQ-9 score, and baseline visual analog scale (VAS) pain score. Secondary hypothesized predictors included history of knee injury, prior HA or ICS injections, location of OA (medial, lateral, patellofemoral, tricompartmental), disability status, smoking history, history of narcotic use, and use of assistive devices (i.e., canes, braces, or walkers).

\subsection{Study Outcome}

The primary outcome was clinical response to therapy, which was defined using established minimal detectable change (MDC) thresholds for the KOOS. The MDC represents the amount of change that must be achieved to reflect a change in clinical status that exceeds the measurement error inherent in a given instrument [15] [16]. The MDC was calculated based on test-retest reliability coefficients [the intra-class correlation coefficient (ICC)] reported in four prior studies using the KOOS score [12] [17] [18] [19]. The MDC at the 95\% confidence interval $\left(\mathrm{MDD}_{95 \%}\right)$ was calculated as follows: $\mathrm{MDD}_{95 \%}=\mathrm{z} * \mathrm{SEM} * \sqrt{2}$ where $\mathrm{z}=1.96$ and $\operatorname{SEM}($ standard error of measurement $)=\sigma_{\text {baseline }} \sqrt{(1-\text { ICC })}$. A clinical responder was defined as a change in total KOOS score (or any one subscale KOOS score) that exceeds the mean MDC (and thus exceeds the measurement error inherent in the KOOS).

\subsection{Statistical Analysis}

A multivariate logistic regression model with forced entry was used to determine which of the hypothesized 
primary clinical variables were predictive of response to therapy. Pairwise t-tests and chi-square tests were used to explore and examine differences in clinical characteristics between responders and non-responders. This study was powered to detect moderate to large changes between responders and non-responders (age $>8$ years, BMI $>5 \mathrm{~kg} / \mathrm{m}^{2}$, VAS pain scale $>3$ points). Based on calculations for age, BMI, and VAS pain scale, and given a two-tailed $\alpha$ of 0.05 and desired power $(1-\beta)$ of 0.8 , the sample size would have needed to be $\geq 52$ subjects.

\section{Results}

\subsection{Patients}

Between March 2012 to March 201361 subjects were enrolled, with five lost to follow-up and one withdrawal. Thus, 55 subjects were included in the study. Of those 55, 35 were classified as clinical responders and 20 were clinical non-responders (Figure 2). Baseline demographic and clinical characteristics are shown in Table 1. The predominant patient population was men (44/55 subjects) of African American descent (35/55), as this is also a common population at the PVAMC. The mean BMI was 32, consistent with obesity. Kellgren scores were variable, with a score of two seen most frequently (42\%) and a score of four seen least frequently (9\%). Nearly all subjects (49/55) had medial compartment OA. Approximately half of the subjects had a history of knee injury and/or previous HA injection. Only 2/55 subjects had radiological meniscal calcinosis.

\subsection{Predictors of Clinical Response}

A pairwise analysis of predictors at three months did not demonstrate any difference between responders and non-responders for PHQ-9 depression score, baseline VAS pain score, age, BMI, or Kellgren score (Figure 3). A multivariate logistic regression model using forced entry for the primary predictor variables did not reveal any statistically significant predictors ( $\mathrm{p}=0.451$ for the overall model) (Table 2). Likewise, additional exploratory multivariate logistic regression models using various combinations of predictors failed to reveal any statistically significant predictors. Analysis of secondary predictors of outcome did not show any difference between responders and non-responders for history of knee injury, prior HA or ICS injection, history of smoking, or location of OA (Table 3).

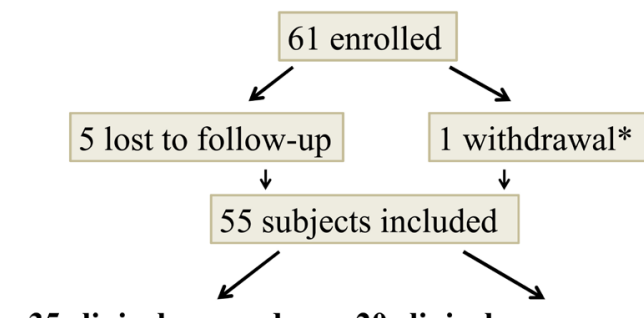

35 clinical responders 20 clinical non-responders

*Expired - not related to viscosupplementation

Figure 2. Study enrollment and follow-up.

Table 1. Baseline characteristics.

\begin{tabular}{cc}
\hline Age (mean \pm SD) & Total $\mathbf{n}=\mathbf{5 5}$ \\
Sex (females/males) (n) & $59 \pm 10.4$ years \\
Race (Caucasian/AA/other) (n) & $11 / 44$ \\
Body mass index (mean \pm SD) & $17 / 35 / 3$ \\
Kellgren score (1/2/3/4) (n) & $32 \pm 7.8$ \\
Medial/Lateral/Tricompartmental (n) & $11 / 23 / 16 / 5$ \\
History of knee injury (yes/no) (n) & $49 / 23 / 16$ \\
Previous HA injection (yes/no/don’t know) (n) & $30 / 25$ \\
Meniscal calcinosis (yes/no) (n) & $24 / 30 / 1$ \\
\hline
\end{tabular}

AA = African American; ${ }^{*} 2$ knee radiographs not done. 


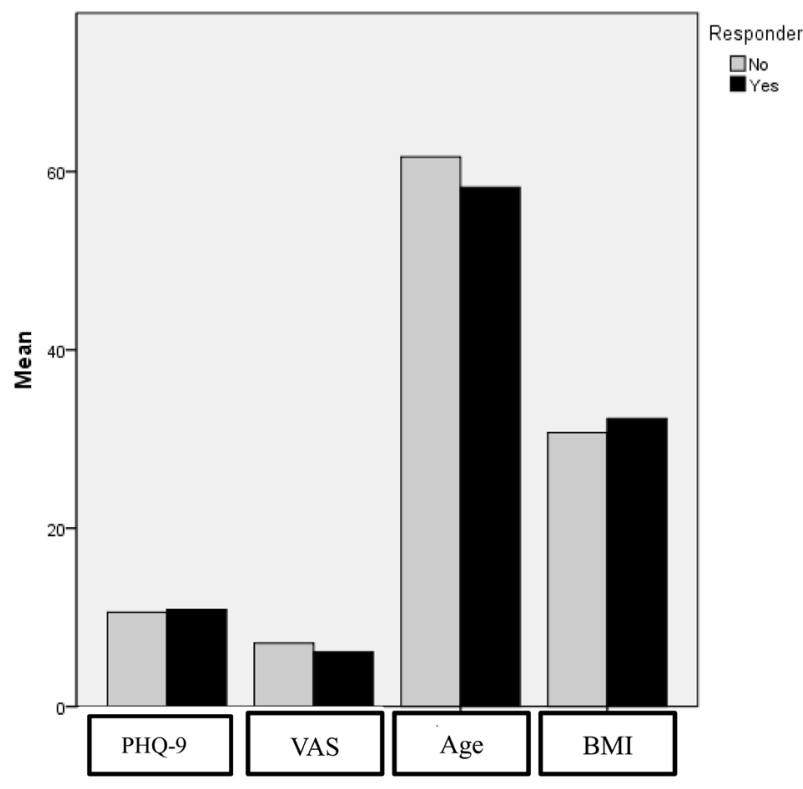

\begin{tabular}{|c|l|l|}
\hline Kellgren & Responder & NR \\
\hline 1 & 8 & 3 \\
\hline 2 & 15 & 8 \\
\hline 3 & 10 & 6 \\
\hline 4 & 2 & 3 \\
\hline
\end{tabular}

Figure 3. Pairwise analysis of predictors at three months. There was no difference between responders and non-responders for any of the hypothesized predictors of response.

Table 2. In the multivariate logistic regression model, none of the primary clinical variables were predictive of clinical response.

\begin{tabular}{cccc}
\hline Predictor & OR & $\mathrm{p}$ & $95 \%$ CI \\
Age & 0.958 & 0.197 & $0.898-1.022$ \\
BMI & 1.029 & 0.530 & $0.941-1.125$ \\
PHQ-9 & 1.037 & 0.984 & $0.947-1.169$ \\
VAS Pain Scale & 0.702 & 0.062 & $0.485-1.1017$ \\
Kellgren $=2$ & 0.677 & 0.731 & $0.142-3.762$ \\
Kellgren $=3$ & 0.676 & 0.781 & $0.119-5.110$ \\
Kellgren $=4$ & 0.529 & 0.582 & $0.037-9.056$ \\
\hline
\end{tabular}

$\mathrm{R}^{2}$ Cox and Snell $=0.133, \mathrm{p}=0.451$; Kellgren scores compared to score of 1 as the reference.

Table 3. None of the secondary variables were predictive of clinical response to the injection.

\begin{tabular}{cccc}
\hline Predictor & Responders (n) & Non-responders (n) & P-value \\
\hline History of knee injury & 15 & 7 & $\mathrm{X} 2=0.317, \mathrm{p}=0.775$ \\
Prior HA injection & 16 & 15 & $\mathrm{X} 2=4.233, \mathrm{p}=0.120$ \\
Previous ICS injection & 32 & 18 & $\mathrm{X} 2=2.336, \mathrm{p}=0.506$ \\
Smoking & 17 & 9 & $\mathrm{X} 2=0.215, \mathrm{p}=0.898$ \\
Medial compartment OA & 31 & 19 & $\mathrm{X} 2=0.636, \mathrm{p}=0.643$ \\
Lateral compartment OA & 13 & 9 & $\mathrm{X} 2=0.823, \mathrm{p}=0.623$ \\
Patellofemoral OA & 26 & 13 & $\mathrm{X} 2=0.826, \mathrm{p}=0.530$ \\
Tricompartmental OA & 10 & 6 & $\mathrm{X} 2=0.013, \mathrm{p}=0.999$ \\
\hline
\end{tabular}

\subsection{Interesting Correlation}

The data were further examined to determine whether there were possible correlations between our hypothesized predictors and changes in total KOOS score. This was thought to represent changes that may have existed, but were perhaps too weak to be revealed in our classification system for defining responders. There was a moderate negative correlation between age and change in total KOOS score at three months after HA injection $(r=-0.34$, $\mathrm{p}=0.01$ ), which implies that increasing age was associated with smaller changes in the total KOOS score at 
three months.

\section{Discussion}

Prior research investing predictors of response to HA in individuals with knee osteoarthritis has been limited by ill-defined criteria for determining clinical response. The present study sought to explore clinical predictors of response to HA by defining response based on established MDC valuesof a valid and reliable outcome measure. Neither Kellgren score, age, BMI, PHQ-9 depression score, nor baseline VAS pain score were found to be factors predictive of clinical response to a single intra-articular injection of Hylan GF-20 at three months. Other predictors, including history of knee injury, prior HA or ICS injections, smoking status, and location of OA were also not predictive of clinical response.

Conrozier et al. found that the presence of a moderate sized effusion, radiological meniscal calcinosis, and joint space loss in a single compartment were predictors of response to viscosupplementation [20]. In our study, the presence of effusion was not evaluated and the latter two factors were not predictive of response. However, the present study only had two subjects with meniscal calcinosis. The discrepant results in the present study, especially with regard to single compartment involvement, may be due to the method for defining response. The present study used an objective measure of outcome, as opposed to Conrozier et al., whose measure of response was a qualitative measure. In that retrospective study, a responder was defined as someone who endorsed a "satisfied" response to HA injection based on a phone interview.

In 2004, a meta-analysis of 20 randomized controlled trails by Wang et al. found that age > 65 and advanced radiographic stage were predictors of clinical non-response [21], neither of which were predictive in our study. The authors defined response using an "efficacy score," which was arrived at using a series of calculations based on pain intensity differences as a function of time. This is a scoring system that is not a standardized, validated measure of outcome, in contrast to our study, which utilized established MDC thresholds for a valid and reliable outcome measure. This ensured that patient-reported changes exceeded test-retest measurement error.

Our study did find a moderate negative correlation between age and change in total KOOS score. Similarly, in Wang's study, older age was found to be a predictor of non-response. Although age was only moderately negatively correlated to change in KOOS score in the present study, there does appear to be some relationship between age and HA response. It would be worthwhile to examine further why older patients have a smaller change in their total KOOS score after three months. Are older patients with OA overall much less active? This is one explanation to account for the little fluctuation in total KOOS score seen in this population three months post-injection.

The strengths of this study include a quantitative measure of outcome and few subjects lost to follow-up at three months. Also, the fact that this was an observational study allowed for assessment of "real world" effects of hyaluronate injections. The observational nature of this study also is a limitation, as there was no control group. There are also other limitations. There was an element of selection bias, as only subjects who were permitted to receive HA injections based on pre-set PVAMC criteria were included. This limited the study population to a more refractory group of individuals, as they had to have failed or been intolerant of other medical therapies. This study was also powered to detect medium to large differences for our predictors, so it is possible for smaller differences to have been missed. However, one can argue whether or not very small differences would even be clinically relevant.

Of note, none of the knee injections were performed using ultrasound guidance. There is evidence that ultrasound guided knee injection improves accuracy, with more likelihood of the injection entering the joint space [22]. Based on this data, it is theoretically possible that a percentage of the HA injections did not enter exactly into the joint space. It would be of interest to investigate whether or not the outcomes would be different using ultrasound guided technique.

\section{Conclusion}

In conclusion, there continue to be no clearly identified clinical predictors to help guide the clinician's decision to administer intra-articular HA. It remains important to determine which patients will benefit most from HA injections, particularly given the high cost associated with these injections. Although the present study did not reveal any factors that predicted efficacy of viscosupplementation in knee OA, it was only powered to detect moderate large changes in predictors. There was a moderate negative correlation between age and change in to- 
tal KOOS score. Future directions would include exploring factors contributing to the relationship between age and response to therapy in a larger sample size, as well as differences in outcome using ultrasound guided HA injections.

\section{Author Contributions}

Study concept and design—Koolaee, Schumacher, Pullman-Mooar Acquisition of data-Koolaee, Pullman-Mooar, Mooar

Analysis and interpretation of data-Koolaee, Schumacher, Pullman-Mooar, Shoemaker

Drafting the article or revising it critically for important intellectual content-Koolaee, Schumacher, Pullman-Mooar, Shoemaker, Mooar

Final approval of the version of the article to be published-Koolaee, Schumacher, Pullman-Mooar, Shoemaker

R. M. Koolaee (michellekoolaee@gmail.com) takes responsibility forthe integrity of the work as a whole.

\section{Conflict of Interest Statement}

The authors declare that they have no conflicting interests.

\section{Role of Funding Source}

Funding for this project was provided by the Penn Center for Musculoskeletal Disorders.

\section{Acknowledgements}

We are grateful to Peter Merkel and Janet Dinnella for their invaluable assistance.

\section{References}

[1] Conduah, A.H., Baker, C.L. and Baker, C.L. (2009) Managing Joint Pain in Osteoarthritis: Safety and Efficacy of Hylan G-F 20. Journal of Pain Research, 2, 87-98.

[2] Brockmeier, S.F. and Shaffer, B.S. (2006) Viscosupplementation Therapy for Osteoarthritis. Sports Medicine \& Arthroscopy Review, 14, 155-162. http://dx.doi.org/10.1097/00132585-200609000-00007

[3] Ghosh, P. (1994) The Role of Hyaluronic Acid (Hyaluronan) in Health and Disease: Interactions with Cells, Cartilage and Components of Synovial Fluid. Clinical and Experimental Rheumatology, 12, 75-82.

[4] Moreland, L.W. (2003) Intra-Articular Hyaluronan (Hyaluronic Acid) and Hylans for the Treatment of Osteoarthritis: Mechanisms of Action. Arthritis Research \& Therapy, 5, 54-67. http://dx.doi.org/10.1186/ar623

[5] Tamoto, K., Tada, M., Shimada, S., Nochi, H. and Mori, Y. (1993) Effects of High-Molecular-Weight Hyaluronates on the Functions of Guinea Pig Polymorphonuclear Leukocytes. Seminars in Arthritis and Rheumatism, 22, 4-8. http://dx.doi.org/10.1016/S0049-0172(10)80014-9

[6] Pozo, M.A., Balazs, E.A. and Belmonte, C. (1997) Reduction of Sensory Responses to Passive Movements of Inflamed Knee Joints by Hylan, a Hyaluronan Derivative. Experimental Brain Research, 116, 3-9. http://dx.doi.org/10.1007/PL00005742

[7] Frampton, J.E. (2010) Hylan G-F 20 Single-Injection Formulation. Drugs \& Aging, 27, 77-85. http://dx.doi.org/10.2165/11203900-000000000-00000

[8] Chevalier, X., Jerosch, J., Goupille, P., van Dijk, N., Luyten, F.P., Scott, D.L., et al. (2010) Single, Intra-Articular Treatment with 6 ml Hylan G-F 20 in Patients with Symptomatic Primary Osteoarthritis of the Knee: A Randomised, Multicentre, Double-Blind, Placebo Controlled Trial. Annals of the Rheumatic Diseases, 69, 113-119. http://dx.doi.org/10.1136/ard.2008.094623

[9] Bellamy, N., Campbell, J., Robinson, V., Gee, T., Bourne, R. and Wells, G. (2006) Viscosupplementation for the Treatment of Osteoarthritis of the Knee. Cochrane Database of Systematic Reviews, 19, CD005321.

[10] Rutjes, A.W., Juni, P., da Costa, B.R., Trelle, S., Nuesch, E. and Reichenbach, S. (2012) Viscosupplementation for Osteoarthritis of the Knee: A Systematic Review and Meta-Analysis. Annals of Internal Medicine, 157, 180-191. http://dx.doi.org/10.7326/0003-4819-157-3-201208070-00473

[11] Kellgren, J.H. and Lawrence, J.S. (1957) Radiological Assessment of Osteo-Arthrosis. Annals of the Rheumatic Diseases, 16, 494-502. http://dx.doi.org/10.1136/ard.16.4.494 
[12] Roos, E.M. and Toksvig-Larsen, S. (2003) Knee Injury and Osteoarthritis Outcome Score (KOOS) —Validation and Comparison to the WOMAC in Total Knee Replacement. Health and Quality of Life Outcomes, 1, 17. http://dx.doi.org/10.1186/1477-7525-1-17

[13] Davis, A.M., Perruccio, A.V., Canizares, M., Hawker, G.A., Roos, E.M., Maillefert, J.F., et al. (2009) Comparative, Validity and Responsiveness of the HOOS-PS and KOOS-PS to the WOMAC Physical Function Subscale in Total Joint Replacement for Osteoarthritis. Osteoarthritis and Cartilage, 17, 843-847. http://dx.doi.org/10.1016/j.joca.2009.01.005

[14] Kroenke, K., Spitzer, R.L. and Williams, J.B. (2001) The PHQ-9: Validity of a Brief Depression Severity Measure. Journal of General Internal Medicine, 16, 606-613. http://dx.doi.org/10.1046/j.1525-1497.2001.016009606.x

[15] Beaton, D.E., Bombardier, C., Katz, J.N., Wright, J.G., Wells, G., Boers, M., et al. (2001) Looking for Important Change/Differences in Studies of Responsiveness. OMERACT MCID Working Group. Outcome Measures in Rheumatology. Minimal Clinically Important Difference. The Journal of Rheumatology, 28, 400-405.

[16] Portney, L.G. and Watkins, M.P. (2008) Foundations of Clinical Research: Applications to Practice. 3rd Edition, Prentice Hall, Upper Saddle River.

[17] Salavati, M., Akhbari, B., Mohammadi, F., Mazaheri, M. and Khorrami, M. (2011) Knee Injury and Osteoarthritis Outcome Score (KOOS); Reliability and Validity in Competitive Athletes after Anterior Cruciate Ligament Reconstruction. Osteoarthritis and Cartilage, 19, 406-410. http://dx.doi.org/10.1016/j.joca.2011.01.010

[18] Bekkers, J.E., de Windt, T.S., Raijmakers, N.J., Dhert, W.J. and Saris, D.B. (2009) Validation of the Knee Injury and Osteoarthritis Outcome Score (KOOS) for the Treatment of Focal Cartilage Lesions. Osteoarthritis and Cartilage, 17, 1434-1439. http://dx.doi.org/10.1016/j.joca.2009.04.019

[19] Roos, E.M., Roos, H.P., Lohmander, L.S., Ekdahl, C. and Beynnon, B.D. (1998) Knee Injury and Osteoarthritis Outcome Score (KOOS) - Development of a Self-Administered Outcome Measure. Journal of Orthopaedic \& Sports Physical Therapy, 28, 88-96. http://dx.doi.org/10.2519/jospt.1998.28.2.88

[20] Conrozier, T., Mathieu, P., Schott, A.M., Laurent, I., Hajri, T., Crozes, P., et al. (2003) Factors Predicting Long-Term Efficacy of Hylan GF-20 Viscosupplementation in Knee Osteoarthritis. Joint Bone Spine, 70, 128-133. http://dx.doi.org/10.1016/S1297-319X(03)00005-8

[21] Wang, C.T., Lin, J., Chang, C.J., Lin, Y.T. and Hou, S.M. (2004) Therapeutic Effects of Hyaluronic Acid on Osteoarthritis of the Knee. A Meta-Analysis of Randomized Controlled Trials. Journal of Bone and Joint Surgery, American, 86-A, 538-545.

[22] Berkoff, D.J., Miller, L.E. and Block, J.E. (2012) Clinical Utility of Ultrasound Guidance for Intra-Articular Knee Injections: A Review. Clinical Interventions in Aging, 7, 89-95. 